\title{
Liquefaction of Lignocelluloses as a Promising Alternative to Petrochemicals
}

\author{
L Asoka Panamgama* \\ Department of Chemistry, University of Ruhuna Matara, Sri Lanka \\ *Corresponding author: Department of Chemistry, Faculty of Science, University of Ruhuna Matara, Sri Lanka
}

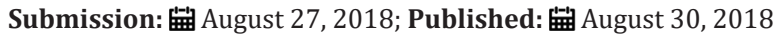

\section{Introduction}

Current world population is heavily dependent on fossil fuels (petrochemicals) for their energy needs and it is difficult to imagine what would happen to the world when this limited resource has come to the end in one day. Fossil resources are limited, environmentally unfriendly and their prices are liable to change unpredictably and rapidly depending on political and economic situations. It has predicted that if we use this scary limited resource at the current rate of consumption, less than a half century it would come to an end. On the other hand, excessive usage of petrochemicals has created a lot of environmental issuers as well, such as polluted environments in most of the highly congested cities in the world and global warming due to greenhouse effect. Further evaluation of much warmer climates in the world is responsible for the rising of sea levels due to melting of snow etc., It can be expected that in the near future tiny island around the globe would be no longer visible and would sink. Therefore, seeking and developing of alternative and new energy resources as well as lowering of the rate of consumption of petrochemicals are equally vital.

Population is also on the rise at an alarming rate in the developing and underdeveloped regions of the globe and as a consequence the corresponding need of petrochemicals is also rapidly growing to fulfil the transportation and other needs. The liquefaction of lignocelluloses is one of the promising methods to produce liquid biofuels and chemicals from the wood and other renewable biomass feedstocks among the thermochemical conversation processes. Different reaction medias such as water organic solvents, and co-solvents with different catalysts such as alkali and catalysis etc., in the presence/absence of a reducing gas such as $\mathrm{H} 2$ and $\mathrm{CO}$ at sub- and super-critical conditions arising from a wide range of temperature and pressure has been used in liquefaction process. Liquefaction of lignocelluloses has been developed as pilot or industrial scales by many researches. Basically, the liquefaction method can be divided into two principle subtitles: solvolysis liquefaction (operated at atmospheric pressure) and pressurized solvolytic liquefaction. Lignocelluloses substances such as wood, bark and other agricultural and non-agricultural materials have played a prominent role in human life throughout history. Wood has been an important construction material since humans began building shelters. Wood has a long history of being used as fuel, which continues even nowadays, mostly in rural areas of the underdeveloped world. They are traditionally used as firewood, build toys, tools, weapons, shelter, house hold furniture, ceiling, flooring materials, transportation and agricultural/ structural materials.

Domestic housing in many parts of the world today is commonly made from timber-framed construction. With time, due to increasing needs with population, deforestation has also increased resulting adverse consequences such as global warming, affecting water cycle, and increasing environmental pollution. The building industry has a large environmental impact due to the extensive use of natural resources as wood. Therefore, increasing awareness considering environmental protection due to decreasing natural resources and climate change has heavily influenced the building sector over the last two decades. This use of petrochemical derived products was found to pollute indoor air quality as well as emit carbon dioxide to the atmosphere causing adverse consequences. Therefore, in recent decades, due to depleting fossil fuel reserves and increasing environmental concerns, researchers and adhesive companies were increasingly interested in developing adhesives from natural resources or incorporating natural polymeric materials such as lignocellulosic biomass into synthetic adhesives. In contrast to fossil fuel resources, plant biomass is a renewable resource.

A waste material bark is low cost plentifully available material. Certain species of bark contains a higher extent of polyphenols and lignin but generally burned as firewood. Recently valuable phenolic compounds and chemicals have extracted from various bark species. Extraction yields, composition of each extracts and thermal properties have been evaluated. Extracts have characterized by wet chemistry viz., Stiasny value, ash content, and several analytical tools viz., FTIR spectroscopy, XRD analysis and thermogravimetric analysis. Heavily dependent production of synthetic adhesives, 
polymers and resins derived from petrochemicals, can be reduced by the usage of lignocellulosic liquid products. Synthetic adhesives are mostly derived from petroleum-based products such as natural gas and petrochemicals specially from crude oil. Adhesive is a material that is used to adhere adherents or laminates including plastics, metal, and wood together by the use of cohesive forces under specified conditions of humidity, temperature, and pressure. They can be classified into two categories as synthetic adhesives and natural adhesives. Synthetic adhesives are used for the production of wood composites such as chipboard, particleboard, fiberboard, oriented standard board, hard board, medium density board etc. Synthetic thermosetting adhesives based on formaldehyde that are widely used in wood construction are amino-formaldehyde adhesives, urea formaldehyde, melamine urea formaldehyde (MUF) and phenolic adhesives as phenol formaldehyde (PF) or phenol resorcinol formaldehyde (PRF).

These synthetic adhesives have advantages like providing strength, durability and enhance the use of wood for construction.
As a result of high formaldehyde and VOC emissions from the wood products engineered in the early stage and reported health impact on people exposed to the formaldehyde and VOC, the industry was forced to lower the formaldehyde content of the traditional adhesives in recent years. Polyurethane (PUR) is a formaldehydefree adhesive introduced for application in wood constructions. Moreover, lower amount of adhesive is required to be applied in wood constructions for PUR, and no additional hardener is necessary. PUR adhesive is expected to cause less environmental damage due to lower amount of adhesive needed and lower amount of elimination of formaldehyde.

In concluding the usage of liquefied products from various waste materials in substitution of petrochemically derived materials will open up new avenues and has a lot of economic benefits. Microwave energy is also a promising alternative energy in addition to the traditional thermochemical energy in conversion of lignocelluloses into liquefied products.
Creative Commons Attribution 4.0 International License

For possible submissions Click Here
Submit Article

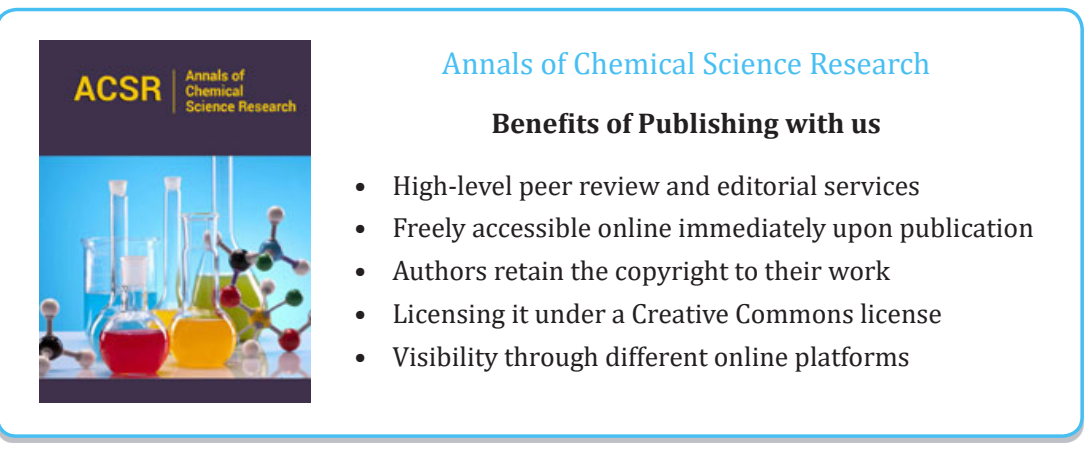

